\title{
Contrast enhanced imaging features of liver metastasis from a meningeal solitary fibrous tumor: a case report
}

\author{
Beilei Lu', Qing Lu, Beijian Huang ${ }^{1,2}$, Haixia Yuan ${ }^{1,2}$, Chaolun Li' \\ ${ }^{1}$ Department of Ultrasound, Zhongshan Hospital, Fudan University, ${ }^{2}$ Shanghai Institute of Medical Imaging, Shanghai, \\ China
}

\begin{abstract}
A solitary fibrous tumor seldom originates from the meninges, and the accuracy of imaging modalities in the diagnosis of meningeal SFT metastasisis is perlexing. Contrast enhanced imaging modalities, such as contrast enhanced ultrasound and contrast enhanced magnetic resonance imaging are valuable in the differential diagnosis of hepatic neoplasms. This case report demonstrated the contrast enhanced ultrasound and enhanced magnetic resonance imaging features of one case of liver metastasis from a menigeal solitary fibrous tumor.

Keywords: solitary fibrous tumor; metastasis; liver; contrast enhanced ultrasound; contrast enhanced magnetic resonance imaging
\end{abstract}

Solitary fibrous tumor (SFT) is a relatively uncommon neoplastic entity originating from mesenchymal cells and seldom affects the central nerve system (CNS). However, SFTs from CNS have a tendency of local recurrence and distant metastasis in bone, lung, kidney, pancreas, adrenal gland, liver, etc [1]. So far, no contrast enhanced ultrasound (CEUS) and rare contrast enhanced magnetic resonance imaging (CE-MRI) features about liver metastasis from menigeal STF have been reported. Thus, we present the contrast enhanced images of SFT liver metastasis in a 60-year-old female.

\section{Case report}

A 60-year-old Chinese woman had a pathologically proved slow-degree malignant meningeal SFT and underwent complete extended resection ten years before presentation in our department. She received postopera-

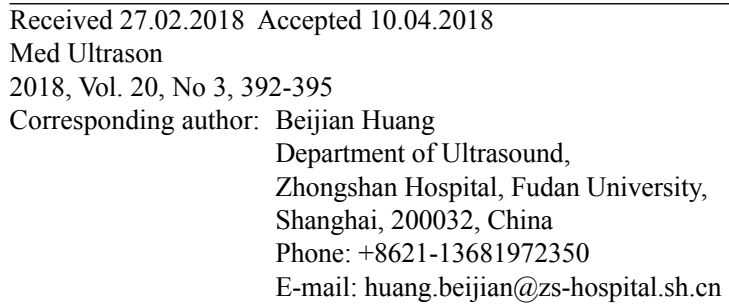

tive radiotherapy and routine follow-up with radiologic brain exams showed no evidence of recurrence. Three months before presenation she underwent CE-MRI for a complaint of back pain and a mass was revealed in the right hepatic lobe with the size $3.0 / 2.7 \mathrm{~cm}$. The lesion showed hypo- and hyper-intense on T1- and T2-weighted images, respectively and homogeneous enhancement in arterial phase without wash-out in portal and delayed phase (fig 1.I). A benign lesion, hepatic angiomyolipoma (HAML) or adenoma (HA) was taken into consideration. Laboratory analysis displayed no elevation of tumor markers, including alpha-fetoprotein, carcinoembryonic antigen, and carbohydrate antigenic 19-9, as well as negative hepatitis $B$ and $C$ viruses.

Meanwhile, as a routine examination for liver lesions in our institution, the patient underwent conventional ultrasounod and CEUS with sulfur hexafluoride microbubbles (Sonovue, Bracco) as contrast agent. A homogeneous hypo-echoic mass with continuous-lined peripheral flow signal was revealed on grey-scale ultrasound (fig 2a,b). The CEUS (GE Logiq E9) enhancement features included: peripheral enhancement at the beginning of arterial phase followed with quick centripetal filling into the center; intense homogeneous enhancement at peak; hyper-enhancement compared with liver parenchyma (no wash-out) in the portal and late phases (fig 3.I). CEUS 


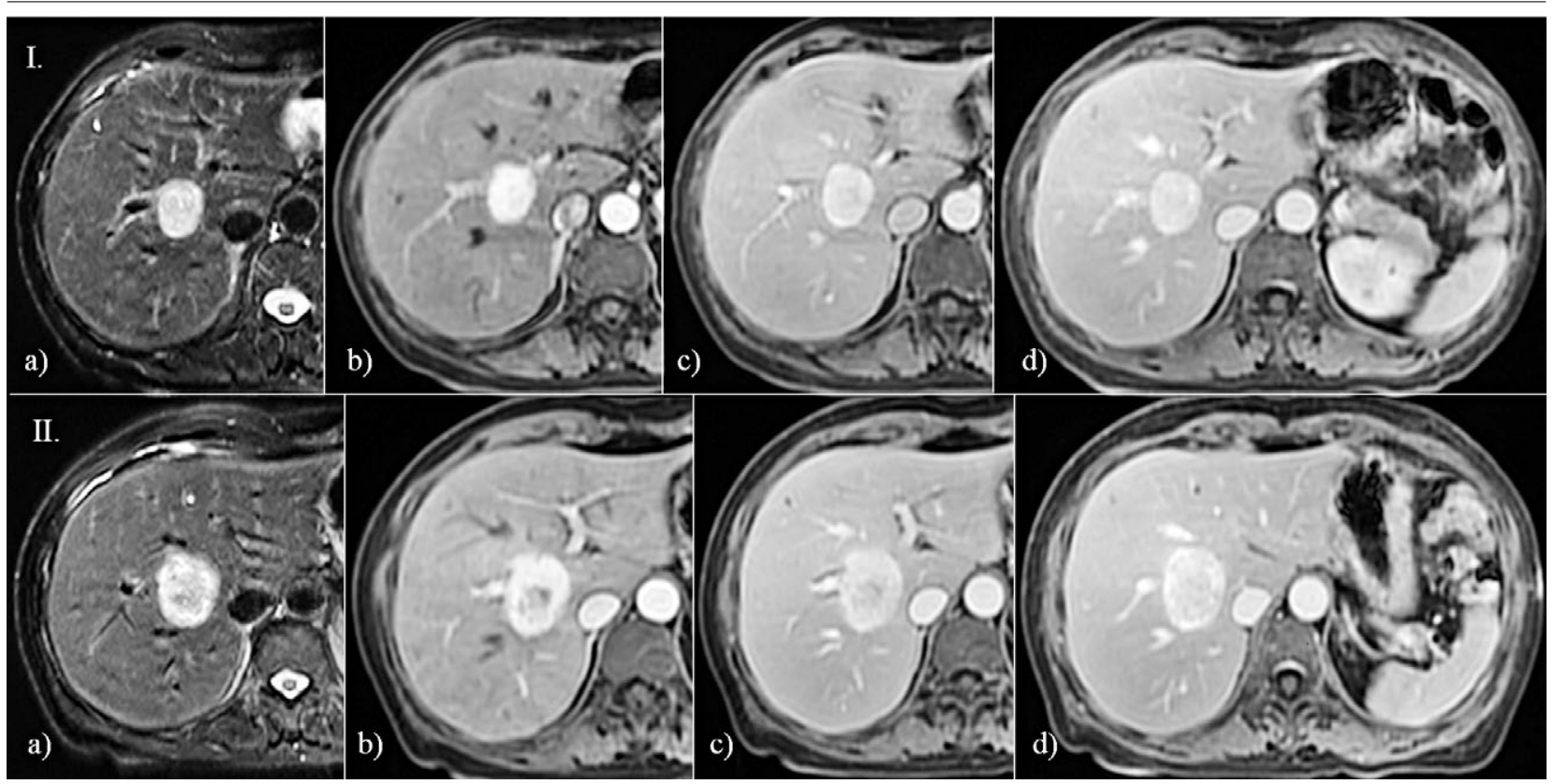

Fig 1. CE-MRI findings of solitary fibrous tumor liver metastasis, I. baseline and II. after 3 months of follow up: a) homogeneous hyper-intense lesion with definite border on T2-weighted image; b) significant homogeneous hyper-enhancement in the arterial phase without wash-out during portal and delayed phases (c,d). Three months later, a larger mass with heterogeneous hyper-intense on T2weighted image was found (a). The lesion showed heterogeneous hyper-enhancement both in the arterial phase (b) and in the portal and delayed phases $(\mathrm{c}, \mathrm{d})$.

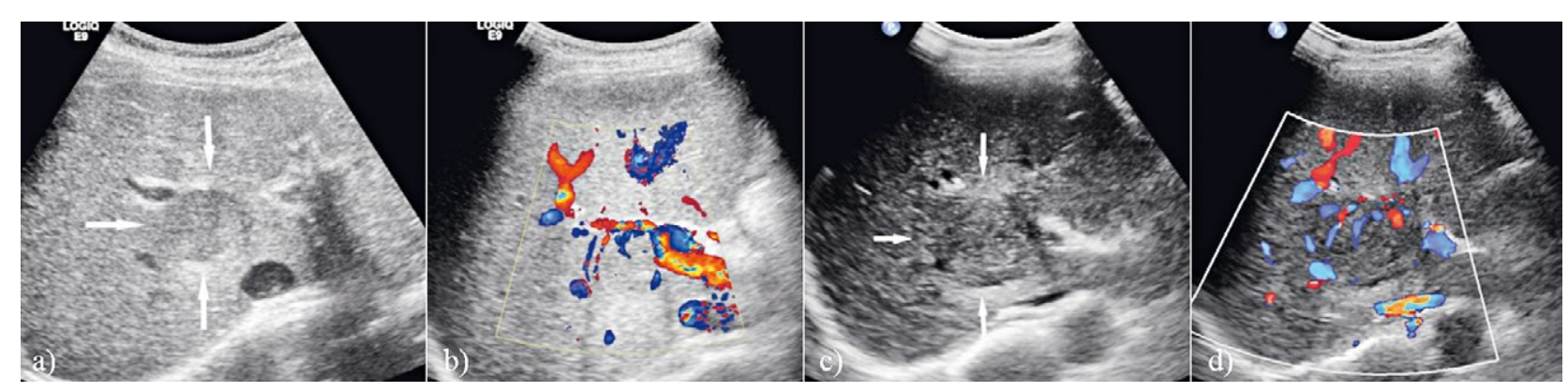

Fig 2. Gray-scale ultrasound revealed ahomogeneous hypo-echoic mass (a) with continuous-lined peripheral flow signal (b). Three months later, the lesion became larger and inhomogeneous with similar peripheral flow signal (c,d).

also drew the diagnosis of a benign liver tumor, a HAML being speculated.

After three months of follow-up CE-MRI found that the liver lesion had increased up to $4.1 / 3.2 \mathrm{~cm}$ and evidenced heterogeneous enhancement during dynamic acquisition (fig 1.II). Conventional ultrasound displayed an inhomogeneous hypo-echoic mass with internal and peripheral flow signal, and also revealed the tumor size increase (fig 2d,c). CEUS (Philips IU Elite) showed peripheral enhancement which rapidly turned into intense hyper-enhancement during the arterial phase. Compared with the previous examination, the peak enhancement was relatively weak, and during portal phase, the en- hancement became more heterogeneous. Furthermore, during the late phase, the central part of the lesion gradually displayed wash-out (hypo-enhancement) (fig 3II). Therefore, a malignant lesion was suspected. The patient underwent right lobe hepatectomy and pathology revealed a lesion composed of spindle cells separated by different amounts of fibrous collagen with heavily central collagenization. Hypercellular areas with low mitotic activity (1mitosis/10HPF) and hemangioperitoidlike structures were also seen. Immunohistochemistry revealed that tumor cells were positive for CD34 and STAT6. The final pathological diagnosis was a metastatic SFT from CNS. 


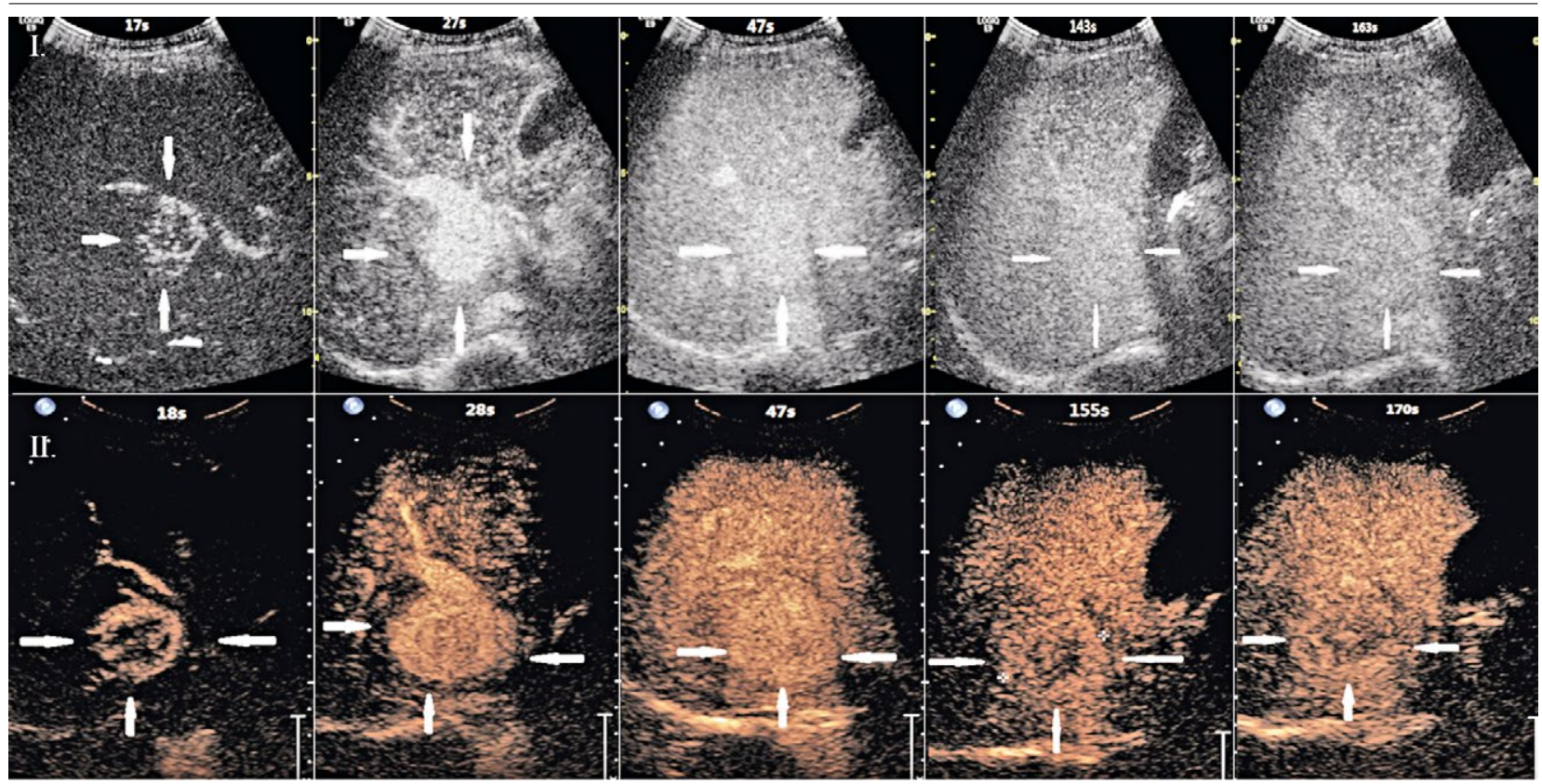

Fig 3. CEUS features of solitary fibrous tumor liver metastasis, I. baseline and II. after 3 months of follow up. During the arterial phase, both showed peripheral with quick centripetal hyper-enhancement, with relative inhomogeneity at peak enhancement after three months. In the portal phase, the images after follow-up showed more inhomogeneity compared with the previous study. In the late phase, the lesion had initially iso-enhancement (no wash-out) comportment and three months later gradual centrifugal wash-out (central hypo-enhancement) was observed.

\section{Discussions}

Recurrence and metastasis of SFT from CNS are regarded as late events. Extra-neural metastases have been reported with an average interval of 8 years after primary treatment [2]. As time went on, the possibility of metastases increases with the 5- and 10-year probability being $4.4-13.0 \%$ and $24.9-33.0 \%$, respectively $[1,3]$. Therefore, along-term follow-up is necessary and more attention should be given when local or distal atypical lesions appear.

In our case report, homogeneous hyper-enhancement without wash-out on both CE-MRI and CEUS induced a diagnosis of benign lesion at the first presentation. However, the lesion size increased more than one centimeter within three months; meanwhile, contrast enhanced images turned out to be heterogeneous, with CEUS even showing hypo-enhancement in the late phase. Together with the history of low-degree malignant meningeal SFT, metastatic SFT was taken into consideration.

The image features and changes may be related to pathological findings. Early peripheral enhancement in the arterial phase may correspond to hyper-vascular tissue with abundant tumor cells at the subcapsular area [4]. With the rapid enlargement of the tumor size, the central tumor cells show a trend to degenerate and atrophy, with the substitution of collagenous fibrous tissue, which may account for heterogeneous hypo-intense and hypo-echoic on T2WI image and gray-scale US. Excessive collagenous tissue can be differentiated from necrosis, hemorrhage, and cystic areas, which would also lead to heterogeneity on imaging. Furthermore, the central collagenous fibrous tissue can also contribute to the inhomogeneous peak enhancement in dynamic CEUS and CE-MRI and the central hypo-enhancement in the CEUS late phase [5-7].

Compared with CE-MRI, CEUS enabled real-time imaging that made possible to find more details and clues for definite diagnosis, such as peripheral hyper-enhancement followed by quick centripetal filling at the beginning of the arterial phase and the centrifugal wash-out in the late phase.

No CEUS feature of liver metastasis from meningeal SFT has ever been reported. Other contrast enhanced image modalities, such as contrasted computed tomography and CE-MRI, have been sparsely applied and reported in primary liver SFT, which displayed peripheral and inhomogeneous enhancement during the arterial phase; more pronounced and centripetal with some non-enhanced areas during the portal phase; finally progressed into persistent and less inhomogeneous during the delayed phase $[6,8-10]$. Our lesion was smaller com- 
pared with previous reported liver SFT metastasis. It is possible that our case would shared the same imaging features if it became larger in size, with more necrosis and degeneration.

In conclusion, compared with CE-MRI,CEUS allows the dynamic detection of enhancement features of the lesion and can offer more diagnostic clues for liver metastasis from meningeal SFTs.

\section{Reference}

1. Kim JH, Jung HW, Kim YS, et al. Meningeal hemangiopericytomas: long-term outcome and biological behavior. Surg Neurol 2003;59:47-53.

2. Koyama H, Harada A, Nakao A, et al. Intracranial hemangiopericytoma with metastasis to the pancreas. Case report and literature review. J Clin Gastroenterol 1997;25:706708 .

3. Guthrie BL, Ebersold MJ, Scheithauer BW, Shaw EG. Meningeal hemangiopericytoma: histopathological features, treatment, and long-term follow-up of 44 cases. Neurosurgery $1989 ; 25: 514-522$.
4. Chen N, Slater K. Solitary fibrous tumour of the liver-report on metastasis and local recurrence of a malignant case and review of literature. World J Surg Oncol 2017;15:27.

5. Fuksbrumer MS, Klimstra D, Panicek DM. Solitary fibrous tumor of the liver: imaging findings. AJR Am J Roentgenol 2000;175:1683-1687.

6. Kandpal H, Sharma R, Gupta SD, Kumar A. Solitary fibrous tumour of the liver: a rare imaging diagnosis using MRI and diffusion-weighted imaging. $\mathrm{Br} \mathrm{J}$ Radiol 2008;81:e282-e286.

7. Moser T, Nogueira TS, Neuville A, et al. Delayed enhancement pattern in a localized fibrous tumor of the liver. AJR Am J Roentgenol 2005; 184:1578-1580.

8. Soussan M, Felden A, Cyrta J, Morère JF, Douard R, Wind P. Case 198: Solitary fibrous tumor of the liver. Radiology 2013;269:304-308.

9. Feng LH, Dong H, Zhu YY, Cong WM. An update on primary hepatic solitary fibrous tumor: An examination of the clinical and pathological features of four case studies and a literature review. Pathol Res Pract 2015;211:911-917.

10. Serter A, Buyukpinarbasili N, Karatepe O, Kocakoc E. An unusual liver mass: primary malignant mesothelioma of the liver: CT and MRI findings and literature review. Jpn J Radiol 2014;33:102-106. 